\title{
DIVERSIDAD DE GÉNERO EN LA MOVILIDAD COTIDIANA EN LA COMUNIDAD DE MADRID'
}

\author{
Juana María Rodríguez Moya y Juan Carlos García Palomares \\ Departamento de Geografía Humana. Universidad Complutense de Madrid
}

\section{RESUMEN}

La movilidad cotidiana crece, se diversifica y afecta al conjunto de la población. Es un fenómeno complejo que influye en la calidad de vida y tiene impactos ambientales y territoriales, por lo que ocupa hoy un lugar central en la planificación. Aquí analizamos las diferencias de género en la movilidad cotidiana de la población en la Comunidad de Madrid, y los condicionantes impuestos por la edad, la actividad y la nacionalidad. Se hace especial mención a las diferencias en las edades de 26 a 40 años, momento del ciclo de la vida cuando los contrastes son mayores, y se introduce un análisis según tipologías de espacios metropolitanos, con el fin de introducir el papel de las características territoriales. Se ha usado los datos de la Encuesta Domiciliaria de Movilidad realizada por el Consorcio de Transportes de Madrid durante el mes de noviembre de 2004 (EDM'04).

Palabras clave: movilidad cotidiana, género, Comunidad de Madrid.

\section{ABSTRACT}

The daily mobility increases and concerns the whole population. It is a complex phenomenon that influences in the quality of life and has environmental and territorial impacts. Here we analyze the differences of gender in the daily mobility in the Community of Madrid. We study the influence of the age, activity and nationality. Special mention is made of differences between the ages of 26 and 40 years, the time of life when the contrasts are greater. Finally, we differentiate types of metropolitan spaces in order to introduce the

Fecha de recepción: junio 2010.

Fecha de aceptación: febrero 2012.

1 Este trabajo ha sido financiado por el proyecto TRA2008-06682/MODAL-Relaciones entre modelo territorial y movilidad metropolitana: análisis y modelización. Los autores agradecen al Consorcio Regional de Transportes de Madrid la información suministrada de la EDM2004. 
paper of the territorial characteristics. We have used the information of Mobility Survey realized by the Consorcio de Transportes de Madrid during November, 2004 (EDM'24).

Key words: daily mobility, gender, Comunidad de Madrid.

\section{INTRODUCCIÓN: CIUDAD DISPERSA, MOVILIDAD Y DIFERENCIAS DE GÉNERO}

La literatura sobre las transformaciones territoriales y sociodemográficas en los espacios metropolitanos en las últimas décadas muestra un proceso de expansión urbana dinámico, complejo, contradictorio, acelerado e 'incontrolado', que define la ciudad dispersa o ciudad difusa (Mella y López, 2006; Gutiérrez y García, 2007a; Méndez, 2007; Méndez y Rodríguez, 2007). Estas transformaciones están caracterizadas por las bajas densidades, la separación, discontinuidad y segregación de usos del suelo, que incrementan la movilidad, en especial aquella que se apoya en un uso intensivo del automóvil. Las piezas dispersas y fragmentadas que configuran la periferia metropolitana están conectadas entre sí a través de una red viaria densa y compleja (Dupuy, 1995), mientras desaparecen las relaciones de proximidad asociadas a la ciudad compacta y los desplazamientos peatonales. Por su parte, los transportes públicos se ven incapaces de servir el nuevo modelo de relaciones, con flujos cada vez más complejos, largos y diversos (Gutiérrez y García, 2007b). Se han creado así unos espacios (aquellos que en la literatura norteamericana se han denominado sprawl urban) muy dependientes del automóvil.

La ciudad dispersa no sólo implica cambios en el territorio, también en los modos de vida de la población, que asociados a la globalización cultural, se traducen en la generalización de un modo de vida suburbano. Se transforman los hábitos de la población, como los que tienen que ver con las pautas de consumo y ocio, ahora vinculados a grandes centros comerciales y de ocio en la periferia, pero también otras actividades de relación (amistad, vecindad, etc.), que dejan de ser de proximidad para pasar a ser a distancia. Todo ello provoca evidentes efectos en la movilidad diaria, en su intensidad, los motivos de desplazamientos o la distancia o el tiempo dedicado a los mismos.

En esta ciudad dispersa la movilidad cotidiana crece, se diversifica y afecta al conjunto de la población, es un fenómeno complejo que influye en la calidad de vida y tiene impactos ambientales y territoriales. Por eso, la movilidad ocupa hoy un lugar central en la planificación. La movilidad diaria expresa a la perfección la síntesis de fenómenos territoriales (organización funcional del espacio y estructura urbana-metropolitana) y sociales (estilos de vida, actividades y espacios cotidianos, división del trabajo en el hogar). Se convierte en un indicador de los procesos de cambio territorial y de la diversidad de situaciones urbanísticas, funcionales y sociodemográficas que están emergiendo en grandes regiones urbanas como la de Madrid (Díaz, Rodríguez y García, 2009).

Pero además, la movilidad de la población está también estrechamente ligada a su caracterización sociodemográfica, económica o cultural, de manera que los cambios que se dan en ella como consecuencia del nuevo modelo territorial disperso y de los nuevos estilos de vida afectan de forma diferente a los distintos grupos de población. Un análisis de género debe incluir una conciencia de cómo el género es constitutivo y trabaja a través de una mirada de 
diversos factores, tales como la formación de identidad, los símbolos y significados culturales, comportamientos sociales y normas y la organización social (Polk, 2004). Son varias las reflexiones que desde la perspectiva de género ha suscitado el hecho de que las mujeres tengan distinta movilidad que los hombres, de manera que podemos advertir cómo se están reproduciendo las desigualdades de clase, raza y etnia entre las mujeres, reproduciéndose además la estructura patriarcal. La capacidad de desplazarse es diferente según el sexo, la edad, el estado civil o el número de hijos e hijas, y también según niveles de estudios, renta o entre los distintos grupos étnicos.

En el caso de las diferencias por género, la nueva configuración urbana es especialmente negativa para las mujeres, en cuanto que sus características alteran de forma significativa el uso de los espacios y el empleo de sus tiempos debido a que la vida cotidiana de las mujeres se encuentra más cargada de actividades. Por ello, «la movilidad espacial ha afectado a las pautas de movilidad de hombres y mujeres, provocando que las personas tengan que realizar un enorme gasto de tiempo, dinero, energía, además de otros costes (ambientales, en accidentes) para desplazarse de un sitio a otro (del hogar al centro de trabajo, a las zonas de ocio, compras, etc.), lo que no es igual para hombres y mujeres (ni personas con discapacidad, inmigrantes, menores de edad, etc.) debido a la multiplicidad de tareas que son asignadas a éstas y a su menor acceso real al transporte motorizado» (Segales, 2007).

La intersección de género y la movilidad puede ser expresada en cómo el género influye en las necesidades de viaje individuales, las actitudes y las valoraciones de la movilidad, así como las estructuras de poder de género y en la planificación del transporte y la política (Ley, 1999). El cruce entre movilidad y género, como perspectiva de análisis, hace visible un conjunto de desigualdades en el acceso a las oportunidades de la vida en la ciudad. Como se señala en la Carta Europea de la Mujer en la Ciudad (1995) «el marco de vida en la ciudad (a nivel del barrio y la vivienda) influye y determina la realidad cotidiana de sus habitantes, hombres y mujeres, pero especialmente a las mujeres porque muchas tienen doble jornada de trabajo y dependen por eso más de la calidad de los servicios urbanos y de las opciones adoptadas para el transporte y el medio ambiente».

La mayoría de los trabajos de investigación llevados a cabo en distintas ciudades occidentales muestran resultados asimétricos en cuanto a los patrones de comportamiento de movilidad cotidiana de hombres y mujeres (Díaz, 1988, 1995; Little, 1994; Sabaté, Rodríguez y Díaz, 1995; Costras, 1997; Law, 1999; Miralles, 1998; Ley, 1999; Cristaldi, 2003; Lee y Mcdonald, 2003). Hay un número relevante de investigaciones que se han centrado en el estudio de los desplazamientos al trabajo (Fagnani, 1977; Maddens, 1981; Howe y O’Connor, 1982; Clos, 1986; Hjorthol, 1998; Salom y Delios 1998; Casado, 2000; Vandermissen, Villeneuve y Theriault, 2005; Hoz, 2008; Díaz, Rodríguez y García, 2009), mientras otros estudios lo han hecho en analizar la influencia del uso de coche a diario (Polk, 2004; Best y Lanzendorf, 2005) comprobándose que existe una menor movilidad por parte de determinados grupos que no pueden acceder al coche, cuando éste es el modo de transporte dominante en las periferias, muchas veces el único. Como han señalado Cebollada y Miralles (2003), aunque la tendencia aparente es a la disminución de las diferencias en las posibilidades de uso del automóvil entre los distintos colectivos sociales, hay elementos que parecen contradecirla. Las diferencias entre hombres y mujeres en la posesión del carné de conducir persisten en todos los grupos de edad y en todos los colectivos profesionales. Avanzando un 
poco más, Hanson (2010) plantea que es necesario relacionar la movilidad y el género con el lugar y con el contexto, ya sea de forma tanto cuantitativa como cualitativa, y así realizar análisis de movilidad sostenible.

Una perspectiva interesante en el análisis de estos procesos se basa en reconocer los factores que influyen en la diferente movilidad cotidiana de hombres y mujeres (Díaz, 1995; Salom y Delios, 1998; Lee y McDonald, 2003; Cristaldi, 2005; Monzón et al., 2008). Estos estudios han constatado ya el efecto de determinadas variables espaciales de la movilidad por género a distintas escalas. Sin embargo, en estos trabajos no se ha insistido en cómo afecta el modelo de ciudad dispersa a las diferencias de género en la movilidad.

Por eso, en este artículo pretendemos abordar la movilidad cotidiana desde una perspectiva de género y territorial, se trata de ver en qué medida los roles de género afectan a la movilidad cotidiana según ámbitos territoriales. Se analiza la movilidad diaria como instrumento para el reconocimiento de los procesos de cambio en los modelos territoriales, a través de los contrastes entre hombres y mujeres en sus desplazamientos. Nos centraremos en el análisis de las diferencias de movilidad cotidiana impuestas por la edad, la actividad y la nacionalidad relacionándolas con la movilidad media a lo largo de distintas dimensiones (distancia y tiempo recorrido, el modo y los motivos de los desplazamientos, ya que son los indicadores con mayor brecha de género). A la vez, se hará especial mención a las diferencias en las edades de 26 a 40 años, momento del ciclo de la vida en el que los contrastes son mayores. Todo ello lo haremos diferenciando tipologías de espacios metropolitanos. Se intenta averiguar el impacto del modelo territorial de ciudad dispersa, presente en determinados espacios del área metropolitana y sobre todo en los municipios periurbanos, en las diferencias de la movilidad según género.

\section{II. ÁMBITO DE ESTUDIO, METODOLOGÍA Y FUENTES}

Durante la segunda mitad del siglo pasado Madrid se configuró como un espacio metropolitano con una gran ciudad principal y ciudades suburbanas en desarrollos compactos y muy densos. Se generó una metrópoli monocéntrica, con desplazamientos radiales periferiacentro, relativamente bien servidos por el transporte público. Sólo los municipios metropolitanos del oeste presentaron una morfología urbana diferente, de vivienda unifamiliar, dispersa, con bajas densidades ocupadas por clases acomodadas, pero también con una fuerte relación con la ciudad central. En la actualidad, es en el periurbano donde se encuentran los espacios más dinámicos. Municipios situados más allá del espacio metropolitano tradicional (a unos 30-40 km de la ciudad central), que crecen como consecuencia de la llegada de población desde las propias ciudades suburbanas, a espacios dispersos y de baja densidad. En estos «territorios del coche», la población puede alejarse cada vez más de la ciudad central porque sus relaciones ya no se dan con ella, sino con los múltiples subcentros de actividades que surgen en la periferia metropolitana, relacionados con los procesos de descentralización (Gutiérrez y García, 2007a).

En este trabajo se toma como ámbito de estudio el conjunto de la Comunidad de Madrid. Para conocer cómo afectan a las diferencias de género en la movilidad las distintas estructuras metropolitanas, en especial las diferencias que se producen entre los espacios metropolitanos tradicionales y los espacios periurbanos, hemos establecido una agrupación de 
los municipios según grandes ámbitos territoriales (Figura 1). Para ello hemos realizado un análisis clúster que incluía variables como el total de población, la dinámica reciente, la proporción de unifamiliares, el crecimiento de unifamiliares y la proporción de vivienda secundaria. Eso nos ha permitido separar la ciudad central y diferenciar los municipios del periurbano (con morfologías ligadas al fenómeno del sprawl) de los municipios del área metropolitana tradicional (separando además los grandes y densos municipios metropolitanos tradicionales de los municipios del oeste metropolitano). En una categoría aparte se han separado una serie de municipios metropolitanos con desarrollos recientes y dinámicas diferentes tanto a los metropolitanos tradicionales como a los periurbanos, como Tres Cantos, Rivas Vaciamadrid, Arganda, etc., que presentan crecimientos recientes elevados pero con una fuerte dependencia de Madrid. Finalmente se establece una categoría de municipios que presentan un marcado carácter rural.

\section{TIPOS DE ESPACIOS EN LA COMUNIDAD DE MADRID}

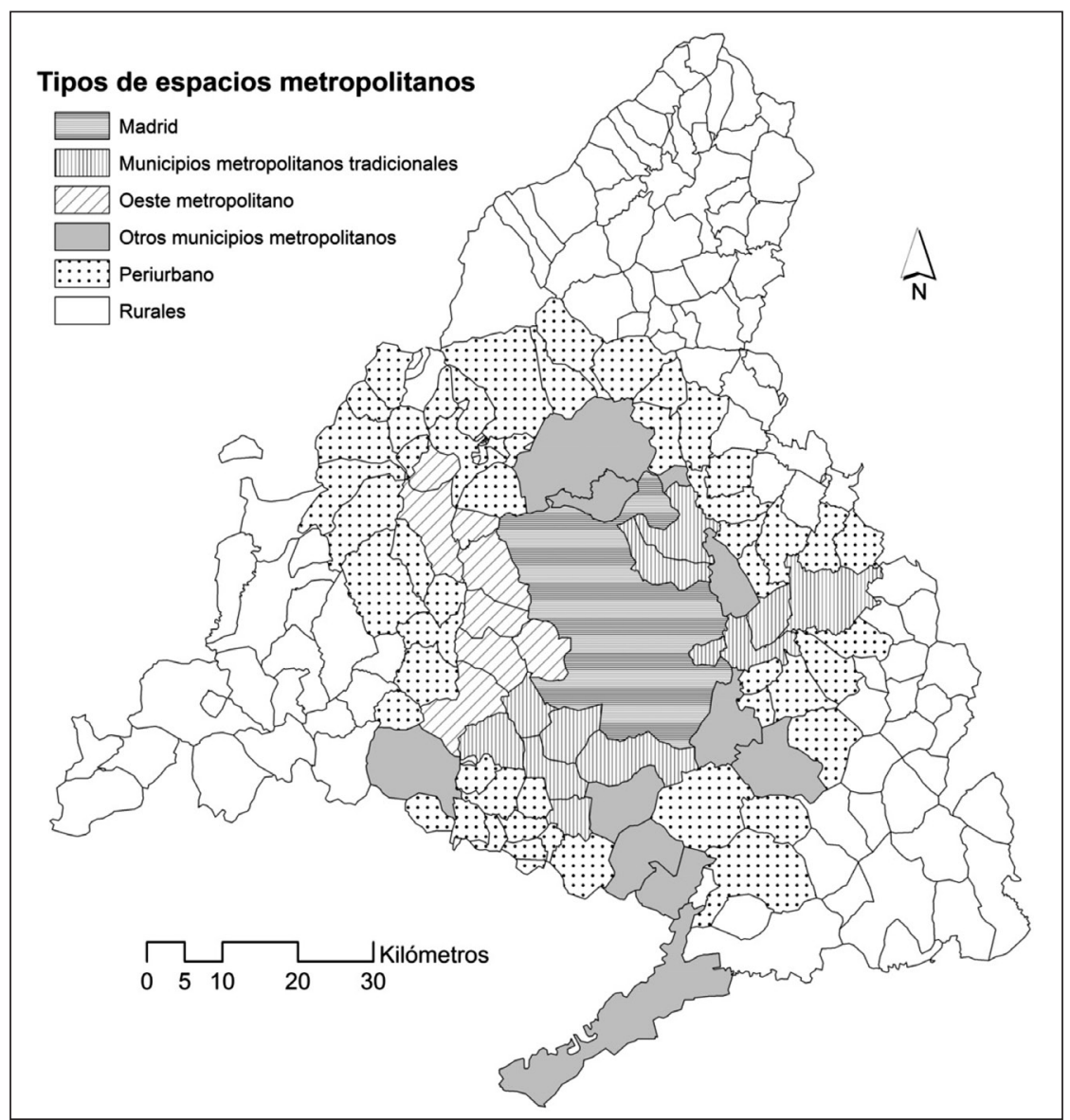

Fuente: Elaboración propia. 
La ciudad de Madrid y los municipios metropolitanos tradicionales se caracterizan por unas densidades altas y una leve recuperación demográfica reciente como consecuencia de la llegada de población extranjera. La principal diferencia se da en los mayores niveles de renta y de actividad en la ciudad central. Frente a estos espacios, los municipios del oeste metropolitano se caracterizan por bajas densidades residenciales, asociadas a niveles de rentas altas, con elevadas tasas de actividad. Es en el oeste donde encontramos las zonas más dinámicas del espacio metropolitano en la actualidad. No obstante, el mayor dinamismo de la región se da en el espacio periurbano, donde está llegando un número relativamente importante de población extranjera pero, sobre todo, población nacional. Se trata de matrimonios jóvenes que buscan espacios residenciales de baja densidad a precios asequibles, de manera que los niveles de renta y las tasas de actividad no alcanzan los valores del oeste metropolitano.

Para el análisis de la movilidad hemos contado con los datos de la Encuesta Domiciliaria de Movilidad realizada por el Consorcio de Transportes de Madrid durante el mes de noviembre de 2004 (EDM'04). La EDM'04 es la última encuesta disponible en la Comunidad de Madrid y con los datos del Censo de 2001 sobre lugar de trabajo y estudio proporciona la información más actualizada disponible en lo referente a la movilidad.

El Consorcio de Transportes ha realizado hasta ahora cinco encuestas, en los años 1974, 1981, 1988, 1996 y 2004. De manera que la periodicidad inicial de 7 años no se ha mantenido en los dos últimos años. A la vez, el ámbito de referencia ha ido ampliándose: las primeras se realizaron únicamente en los municipios metropolitanos, mientras las dos últimas tienen como ámbito espacial de referencia el conjunto de la Comunidad de Madrid. La información se recoge a nivel de zona de transporte (1172 zonas en la EDM'2004), correspondiendo las zonas más grandes con la división administrativa del municipio. En la última encuesta se tomó también información de movilidad de las zonas limítrofes a Madrid de Guadalajara y Toledo, que aquí se han desechado al carecer de significación estadística.

En total, en la Comunidad de Madrid la EDM'04 encuestó 34.942 familias. Se recoge la información de movilidad de todos los mayores de 4 años de cada uno de los domicilios encuestados, pidiendo a cada uno de ellos que describa todos los desplazamientos realizados durante un día laborable del mes de noviembre. La estructura del cuestionario permite cruzar los desplazamientos con las variables sociodemográficas, como el sexo, pero también ésta con la edad, actividad o nacionalidad. Aunque con la información recogida podría analizarse la movilidad multi-motivo o las cadenas de viajes y los prismas espacio temporales propuestos en su momento por Hägerstrand (de gran interés en los estudios de género), la forma como el Consorcio de Transportes explota esta información según motivos y la falta de disponibilidad de datos individuales (secreto estadístico) impide este tipo de análisis.

Con objeto de lograr un resultado más conciso que sintetice la relación entre hombres y mujeres, en algunos casos se ha calculado la brecha de género, que representa el porcentaje de desplazamientos de mujeres menos el porcentaje de desplazamientos de hombres. De manera que valoraciones negativas significan porcentajes mayores en hombres que en mujeres, mientras que valores positivos significa que las mujeres tienen mayor presencia. Toda la información ha sido tratada en un programa de base de datos. 


\section{UNA MOVILIDAD DIFERENTE SEGÚN GÉNERO: IMPORTANCIA DE LAS CARÁCTERÍSTI- CAS SOCIODEMOGRÁFICAS Y LA ESFERA REPRODUCTIVA}

El contexto cultural, social y económico es esencial para entender los significados y las prácticas de género. En la Comunidad de Madrid, aunque las diferencias en la movilidad entre hombres y mujeres se han ido acortando en los últimos años (García y Rodríguez, 2008), todavía son significativas tanto en el número de desplazamientos que realizan, como en los motivos por los que se desplazan, los modos que utilizan o las distancias y los tiempos que dedican a esos desplazamientos. Además, la edad, la actividad o la nacionalidad influyen de manera significativa en las pautas de movilidad cotidiana adoptadas por la población.

Las relaciones de género explican que, en general, las mujeres no sólo realizan un número mayor de desplazamientos y por más motivos, también diversifican más los modos de transporte que utilizan, usando mucho menos el coche privado. Mientras entre los hombres el coche es el modo de desplazamiento predominante, las mujeres se desplazan más a pie o en transporte público. Este menor uso del transporte privado por parte de las mujeres responde, por un lado, a diferencias salariales entre hombres y mujeres y en consecuencia a la menor solvencia económica de las mujeres, que no les permite disponer de vehículo privado propio. Por otro lado, cuando en una familia hay un solo coche, es el hombre quien lo utiliza a diario (Sánchez, 2004). Finalmente existe también una mayor concienciación medioambiental en los desplazamientos femeninos, en especial en aquellos desplazamientos no productivos, donde las mujeres se desplazan más en medios sostenibles (Polk, 2003 y 2004; Hanson, 2010).

\section{El papel de la edad}

Las mujeres no forman un colectivo uniforme. De hecho, existen mayores diferencias sociodemográficas entre las mujeres que entre los hombres. El ciclo vital de las mujeres, por ejemplo, es más complicado que el de los hombres (Díaz y Jiménez, 2003), lo que repercute en diferencias en la movilidad que pueden analizarse según grupos de edad² (Tabla 1).

La etapa de la vida en que existen menos diferencias entre hombres y mujeres es en la infancia. Hasta los 17 años los movimientos de niñas y niños se realizan en el espacio local (el barrio) y se organizan en el entorno inmediato a la vivienda. Los desplazamientos son fundamentalmente a pie, seguidos por desplazamientos en coche, pues necesitan ser acompañados por adultos (normalmente sus madres) para ir al colegio, actividades extraescolares, médico, etc. Entre los 18 y 25 años se incrementan los desplazamientos fuera del barrio y diversifican las actividades diarias, crecen los modos mecanizados. El uso del transporte público es mayoritario entre mujeres y hombres jóvenes, pero empiezan a aparecer diferencias.

Las mujeres de 26 a 50 años son las que más usan el transporte privado como modo de desplazamiento. La incorporación de las mujeres al mercado de trabajo en las últimas décadas, junto al aumento del nivel de vida de la población ha permitido que las mujeres de clase media y alta tengan más fácil acceso a un segundo coche en el hogar. Además, la dispersión

2 La explotación de los datos de la EDM'04 por edades puede realizarse a partir de grupos de edad quinquenales. Aquí hemos optado por una división más amplia, intentando establecer grandes grupos que representen el ciclo de vida de las mujeres. 
de los servicios de salud, educativos, deportivos, etc., y la comodidad del coche hace que su uso sea mayor. El coche las permite realizar mayor número de desplazamientos en menor tiempo y poder atender como acompañantes y conductoras a las diferentes actividades que realizan sus hijos e hijas a lo largo del día.

De 50 a 65 años las tasas de actividad descienden significativamente, el trabajo reproductivo es menor y las mujeres vuelven a desplazarse fundamentalmente a pie y en transporte público. Sólo a partir de los 65 años es cuando hombres y mujeres se acercan en proporciones similares a desplazarse a pie o en transporte público.

Es de gran interés conocer cuáles son las diferencias en los modos de transporte utilizados entre hombres y mujeres en los grupos de edad entre 26 y 40 años. Entre la población de 26 y 30 años las diferencias entre hombres y mujeres no son tan marcadas, debido a la prolongación de la educación reglada junto a la progresiva incorporación de las mujeres al mercado de trabajo. Las diferencias se acrecientan de manera muy llamativa entre los 31 y los 40 años, por coincidir con la edad media de acceso al matrimonio (31 años entre las mujeres y 32,5 entre los hombres) y la maternidad más tardía. Así, podemos observar como a pesar de que se incrementa entre las mujeres el uso del coche entre los 31 a 40, crece la brecha con los hombres. Mientras, se reducen las diferencias en el uso del transporte público. Por el contrario, los desplazamientos a pie pasan de representar un 19,3\% entre las más jóvenes a un 32,4\% en las de 31 a 40 años, cuando en los hombres se mantiene constantes y son mucho más reducidos (11,2 y $12,9 \%$ respectivamente). Este desigual reparto modal según edades parece mostrar como las responsabilidades domésticas y de cuidado se asocian con una movilidad peatonal, pues son desplazamientos cercanos al hogar, pero también a un mayor uso del coche, sobre todo entre aquellas que combinan desplazamientos laborales y trabajos reproductivos. A pesar de todo, los hombres hacen un uso más intensivo del coche en estas edades. Por otro lado, las mujeres, al mismo tiempo que se desplazan más andando o en transporte público, suelen ir cargadas con las bolsas de la compra y utensilios escolares de los hijos e hijas, lo que repercute en su salud.

TABLA 1

MODO DE LOS DESPLAZAMIENTOS SEGÚN EDADES Y GÉNERO, 2004 (PORCENTAJES).

\begin{tabular}{|l|r|r|r|r|r|r|r|r|}
\hline Modo & \multicolumn{2}{|c|}{ A pie } & \multicolumn{2}{c|}{ Transporte público } & \multicolumn{2}{c|}{ Coche } & \multicolumn{2}{c|}{ Otros modos } \\
\hline \multicolumn{1}{|c|}{ Edad } & Hombres & \multicolumn{1}{c|}{ Mujeres } & \multicolumn{1}{c|}{ Hombres } & \multicolumn{1}{c|}{ Mujeres } & \multicolumn{1}{c|}{ Hombres } & \multicolumn{1}{c|}{ Mujeres } & Hombres & \multicolumn{1}{c|}{ Mujeres } \\
\hline $4-17$ & 58.00 & 56.21 & 12.69 & 13.09 & 23.22 & 24.32 & 6.09 & 6.39 \\
\hline $18-25$ & 16.23 & 20.90 & 47.47 & 58.49 & 33.76 & 19.35 & 2.54 & 1.27 \\
\hline $26-30$ & 11.17 & 19.26 & 36.81 & 45.89 & 47.81 & 32.96 & 4.20 & 1.89 \\
\hline $31-40$ & 12.94 & 32.45 & 27.23 & 29.04 & 55.48 & 37.03 & 4.35 & 1.48 \\
\hline $41-50$ & 15.38 & 33.62 & 21.91 & 29.61 & 58.12 & 35.11 & 4.59 & 1.67 \\
\hline $51-64$ & 19.92 & 40.91 & 26.25 & 36.94 & 49.63 & 20.42 & 4.20 & 1.73 \\
\hline 65 y + & 44.60 & 51.36 & 36.65 & 36.59 & 16.45 & 8.82 & 2.29 & 3.22 \\
\hline Total & 25.28 & 36.23 & 28.57 & 34.24 & 41.98 & 27.17 & 4.17 & 2.36 \\
\hline
\end{tabular}

Fuente: Elaboración propia a partir de la EDM de 2004. 
Efectivamente, la movilidad de las mujeres es más compleja y diversificada, cargada de numerosas actividades por su creciente participación en el mercado laboral y por seguir recayendo sobre ellas las responsabilidades domésticas y de cuidados, en las que los hombres apenas se han involucrado. De los algo más de 14.511.000 desplazamientos diarios que se realizan en la Comunidad de Madrid según la EDM de 2004, el 53\% lo realizan mujeres, con un ratio de 2,5 desplazamientos diarios, superior a los 2,3 de los hombres. Esta diferencia es todavía mayor en el grupo de edad entre los 30 y los 40 años, donde las mujeres realizan el $59,3 \%$ de los desplazamientos de esa franja de edad, con ratios de 3,4 desplazamientos por mujer frente a 2,3 en los hombres.

Una movilidad más compleja en las mujeres que se refleja en la distribución de desplazamientos según motivos. Entre los hombres las pautas de desplazamiento tienden a ser de tipo pendular (casa-trabajo o casa-estudio) (Díaz, 1988), con más del 67\% de sus desplazamientos por motivo trabajo o estudio. Sin embargo, entre las mujeres, los desplazamientos pendulares representan apenas un $48 \%$, mientras los realizados a compras y por otras tareas reproductivas $^{3}$ son muy superiores entre las mujeres (Figura 2).

Es nuevamente importante observar las diferencias en los grupos de población entre 26 y 40 años. Así, la brecha de género en los desplazamientos al trabajo pasa de $-8,8 \%$ entre 26 a 30 años a $-26,6 \%$ entre 31 y 40 años. Por el contrario, entre las mujeres de 31 a 40 años crecen los desplazamientos a compras y sobre todo aquellos vinculados a acompañar a otras personas y a otros asuntos personales, claramente relacionados con los trabajos reproductivos (Figura 2). Lo que corrobora como el acceso al matrimonio y la maternidad repercuten notablemente en las diferencias de movilidad.

Figura 2

MOTIVOS DE DESPLAZAMIENTOS SEGÚN EDAD Y GÉNERO, 2004 (PORCENTAJES)

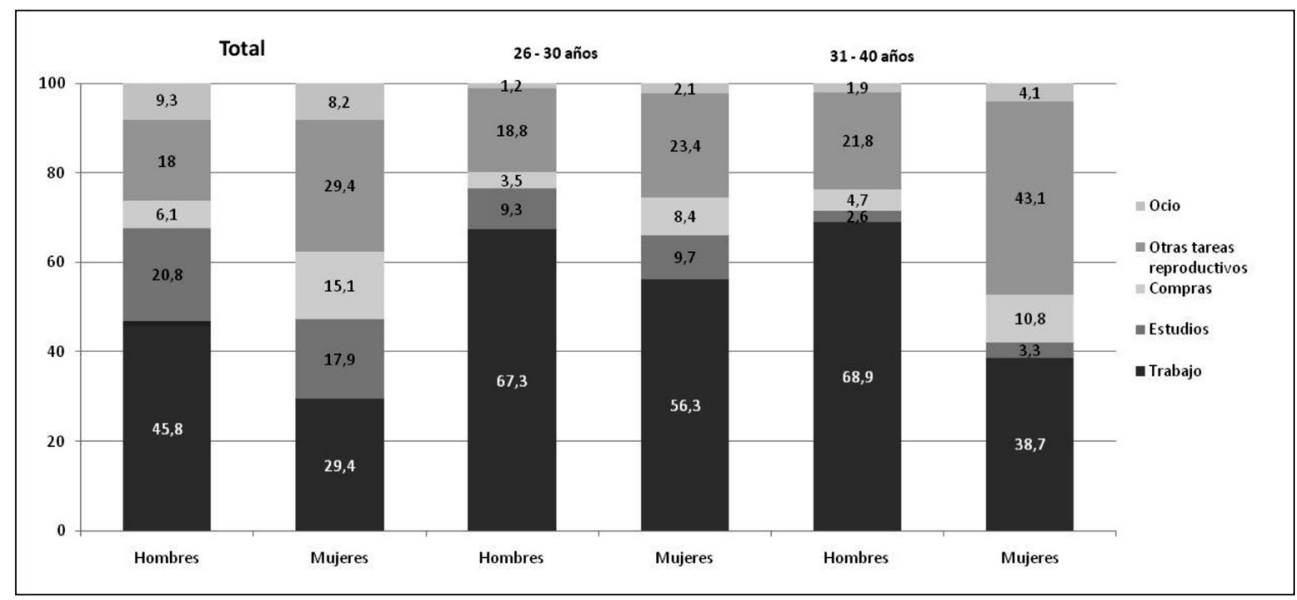

Fuente: Elaboración propia a partir de la EDM de 2004.

3 Dentro de desplazamientos a otras tareas reproductivas se incluyen los realizados al médico, a acompañar a otras personas (en muchos casos dependientes), por gestiones administrativas relativas al hogar o por otras tareas domésticas. 
La edad también introduce diferencias en el tiempo medio de los desplazamientos (Figura 3). Con excepción de la infancia y la adolescencia cuando los tiempos de desplazamientos son similares, y la juventud, cuando las mujeres utilizan más tiempo que los hombres (por el mayor uso del transporte público), en el resto de los grupos de edad los hombres emplean más tiempo que las mujeres. Las diferencias más acusadas se alcanzan entre los 31 y 40 años, coincidiendo con la etapa de la vida en que el trabajo reproductivo de las mujeres las obliga a realizar trayectos más cortos.

TIEMPOS MEDIOS DE LOS DESPLAZAMIENTOS SEGÚN EDADES Y GÉNERO (MINUTOS)

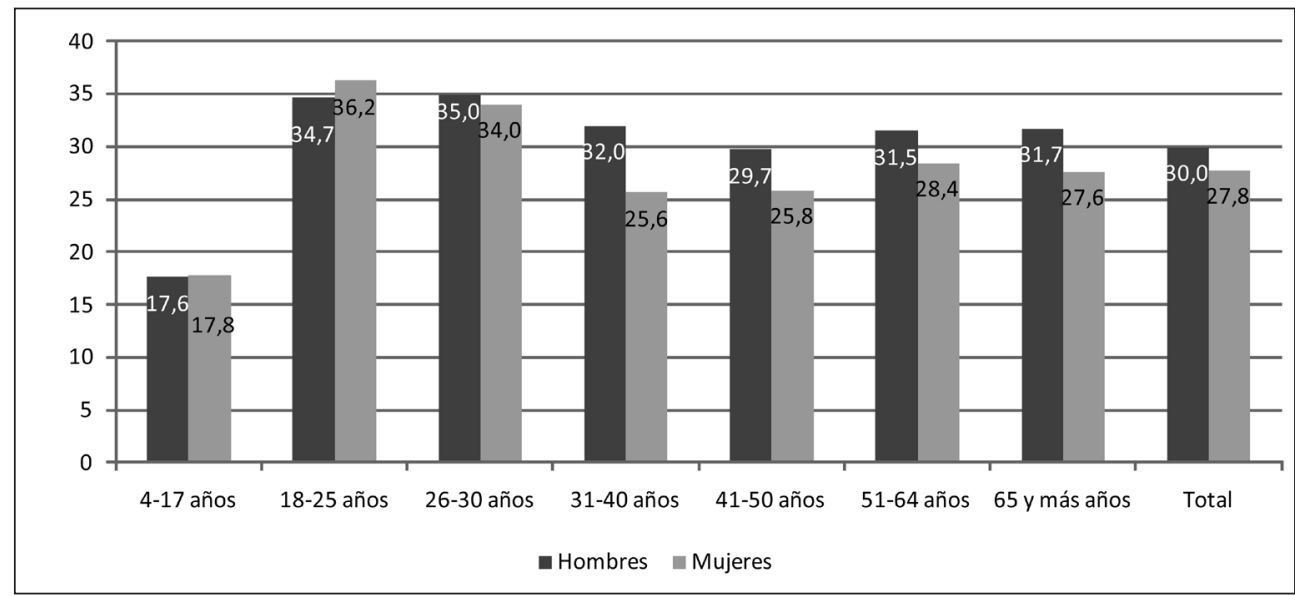

Fuente: Elaboración propia a partir de la EDM de 2004.

Los tiempos de desplazamientos dependen del modo de transporte utilizado (Tabla 2). Los desplazamientos de las mujeres son más cortos en todos los modos, pero destacan las diferencias especialmente en los desplazamientos de las mujeres que usan el coche. Así, los tiempos de los desplazamientos andando son más cortos entre las mujeres de 31 a 40 años (hasta un 15\%), pero estas diferencias aumentan cuando se usa coche ( 7 minutos más cortos de media con los de los hombres, un $23 \%$ ). Esto muestra que en estas edades las mujeres que tienen acceso al coche lo usan para encadenar más desplazamientos, pero más cortos.

Tabla 2

TIEMPOS DE DESPLAZAMIENTO SEGÚN MODO DE TRANSPORTE, EDAD Y GÉNERO (MINUTOS)

\begin{tabular}{|l|c|c|c|c|c|c|}
\hline & \multicolumn{2}{|c|}{ Total } & \multicolumn{2}{c|}{ 26-30 años } & \multicolumn{2}{c|}{ 31-40 años } \\
\hline Modo & Hombres & Mujeres & Hombres & Mujeres & Hombres & Mujeres \\
\hline A pie & 17,1 & 15,1 & 15,2 & 15,0 & 15,8 & 13,4 \\
\hline Transporte Público & 45,2 & 44,2 & 49,8 & 47,9 & 47,7 & 44,7 \\
\hline Coche & 26,9 & 22,2 & 28,8 & 26,2 & 28,1 & 21,4 \\
\hline Otros & 30,9 & 30,4 & 28,0 & 28,0 & 31,6 & 27,4 \\
\hline
\end{tabular}

Fuente: Elaboración propia a partir de la EDM de 2004 


\section{La diferente movilidad según actividad}

Con la incorporación de las mujeres al mercado de trabajo desde mediados de los años ochenta la tasa de actividad femenina en la Comunidad de Madrid pasó del 27\% en 1986 a superar el 50\% en 2001(Rodríguez y Díaz, 2007). Esto ha hecho que la movilidad de las mujeres sea cada día más compleja, modificando la organización de su vida cotidiana.

La actividad afecta muy significativamente a los desplazamientos según género en el reparto según motivos (Tabla 3). Las personas con empleo realizan una proporción muy elevada de desplazamientos laborales, pero como las mujeres siguen manteniendo su rol tradicional de cuidadoras, la proporción de desplazamientos por motivos de compras es el doble entre las mujeres con trabajo remunerado que entre los hombres en la misma situación. De igual forma, la proporción de los desplazamientos que realizan las mujeres relacionados con su papel de cuidadoras es también muy superior al de los hombres.

Son significativas las diferencias entre hombres y mujeres en paro. Un $20 \%$ de los desplazamientos de los hombres en paro es por motivo trabajo (en principio desplazamientos por búsqueda de trabajo) frente al $9 \%$ de las mujeres. Y también es mucho mayor entre los parados que en las paradas el porcentaje de desplazamientos por ocio y deporte. Estas diferencias se mantienen entre los hombres y mujeres que trabajan en labores del hogar, ellos tienen porcentajes de desplazamientos por estudios o por ocio y deportes mucho mayores que ellas, que dedican, por el contrario, una proporción mayor de sus desplazamientos a compras y a desplazamientos relacionados con las tareas reproductivas.

Tabla 3

MOTIVO DE LOS DESPLAZAMIENTOS SEGÚN TIPOS DE ACTIVIDAD Y GÉNERO (PORCENTAJES)

\begin{tabular}{|l|r|r|r|r|r|r|r|r|r|r|}
\hline \multirow{2}{*}{ Motivo } & \multicolumn{2}{|c|}{ Trabajo } & \multicolumn{2}{c|}{ Estudios } & \multicolumn{2}{c|}{ Compras } & \multicolumn{2}{c|}{ Ocio y deporte } & \multicolumn{2}{c|}{$\begin{array}{c}\text { Otros motivos } \\
\text { reproductivos }\end{array}$} \\
\hline \multicolumn{1}{|c}{ Actividad } & Homb. & \multicolumn{1}{|c|}{ Muj. } & Homb. & Muj. & Homb. & Muj. & Homb. & Muj. & Homb. & Muj. \\
\hline Ocupado & 73,7 & 61,6 & 1,9 & 2,9 & 4,1 & 8,5 & 5 & 5,1 & 15,4 & 22 \\
\hline Ocupado y estudia & 46,1 & 42,6 & 36,3 & 32,6 & 1,8 & 3,4 & 6,6 & 6,9 & 9,3 & 14,5 \\
\hline $\begin{array}{l}\text { Retirado/Pensionista } \\
\text { /ncapacitado }\end{array}$ & 2,2 & 0,9 & 0,9 & 2,8 & 23,2 & 32,2 & 28,2 & 18,2 & 45,5 & 45,9 \\
\hline Parado & 20 & 9 & 14 & 8,8 & 12,3 & 20,2 & 14,1 & 7,4 & 39,6 & 54,7 \\
\hline Estudiante & 0,3 & 0,5 & 79,7 & 77,7 & 1,7 & 3,7 & 10,2 & 8,6 & 8,2 & 9,6 \\
\hline Labores del hogar & 0,4 & 0,5 & 11,4 & 2,6 & 29,4 & 33,4 & 18,1 & 11,9 & 40,7 & 51,6 \\
\hline Otras situaciones & 24,8 & 27,2 & 16,6 & 9,4 & 11,2 & 21,1 & 6,2 & 4,7 & 41,2 & 37,5 \\
\hline Total & 45,8 & 29,4 & 20,8 & 17,9 & 6,1 & 15,1 & 9,3 & 8,2 & 18 & 29,4 \\
\hline
\end{tabular}

Fuente: Elaboración propia a partir de la EDM de 2004

Aparecen también diferencias en el reparto modal (Tabla 4). Las mujeres ocupadas se desplazan fundamentalmente en transporte público (41\%) y a pie $(22 \%)$ para ir al trabajo remunerado, mientras los hombres lo hacen mayoritariamente en vehículo privado $(55 \%)$. 
En el caso de las mujeres que trabajan en las labores del hogar, más de la mitad de los desplazamientos se realizan a pie. El barrio de residencia constituye el espacio de referencia en el funcionamiento cotidiano del hogar. Las mismas diferencias por género que aparecen según motivos vuelven a encontrarse en el modo de desplazamiento entre las mujeres y hombres en paro. Ellos usan mucho más los modos mecanizados (tanto transporte público como privado) mientras ellas se desplazan más andando. Eso se traduce en mercados laborales mucho más amplios espacialmente entre los hombres que entre las mujeres.

Efectivamente, en estudios previos se ha argumentado que las mujeres buscan empleos cerca del hogar para poder compatibilizarlo con las actividades domésticas, por no disponer de coche o porque su disponibilidad está condicionada a que no lo use el hombre de la unidad familiar (Fagnani, 1986; Hjorthol, 1998; Casado, 2000). Sin embargo, cada vez más las mujeres tienen mayor dificultad para encontrar empleos cerca del lugar de residencia y están obligadas a utilizar transporte público para desplazarse. Este cambio puede estar relacionado, por un lado, con el desarrollo urbanístico actual, disperso y fragmentado en la Comunidad de Madrid, y por otro, con la creciente inmigración, ya que las mujeres inmigrantes se ven obligadas a recorrer mayores distancias entre el lugar de residencia y el lugar de trabajo.

Tabla 4

MODO DE TRANSPORTE SEGÚN TIPO DE ACTIVIDAD Y GÉNERO, 2004 (PORCENTAJE)

\begin{tabular}{|l|r|r|r|r|r|r|r|r|}
\hline \multicolumn{1}{|c|}{ Modo } & \multicolumn{2}{|c|}{ A pie } & \multicolumn{2}{c|}{ Transporte público } & \multicolumn{2}{c|}{ Coche } & \multicolumn{2}{c|}{ Otros modos } \\
\hline \multicolumn{1}{|c}{ Actividad } & Hombres & Mujeres & Hombres & Mujeres & Hombres & Mujeres & Hombres & Mujeres \\
\hline Ocupado & 13,3 & 21,6 & 27,4 & 40,7 & 54,9 & 35,8 & 4,5 & 1,9 \\
\hline Ocupado y estudia & 13,3 & 20,6 & 43,4 & 56,6 & 41,2 & 21,3 & 2,1 & 1,6 \\
\hline $\begin{array}{l}\text { Retirado/Pensionista/ } \\
\text { Incapacitado }\end{array}$ & 44,6 & 48,1 & 34,7 & 37,7 & 18,8 & 11,2 & 1,9 & 2,9 \\
\hline Parado & 29,3 & 42,1 & 37,3 & 29,7 & 31,3 & 26,8 & 2,1 & 1,5 \\
\hline Estudiante & 46,4 & 45 & 26,1 & 29,7 & 22,6 & 20,7 & 4,9 & 4,7 \\
\hline Labores del hogar & 51,4 & 55,3 & 28,7 & 23 & 18,1 & 20,3 & 1,8 & 1,4 \\
\hline Otras situaciones & 23,1 & 31 & 35,3 & 44,4 & 22,8 & 11,2 & 18,8 & 13,4 \\
\hline Total & 25,3 & 36,2 & 28,6 & 34,2 & 42 & 27,2 & 4,2 & 2,4 \\
\hline
\end{tabular}

Fuente: Elaboración propia a partir de la EDM de 2004.

Como vimos, la distribución de los desplazamientos según el tiempo medio revelaba que los hombres emplean más tiempo en sus desplazamientos que las mujeres, pero si desagregamos esos tiempos según actividad se comprueba que, en general, las mayores brechas aparecen entre las mujeres y hombres parados $(-6,6)$ y los retirados $(-3,1)$. Sin embargo, entre las personas que trabajan y estudian las mujeres emplean más tiempo medio que los hombres (Figura 4). El mayor uso del transporte público explica los mayores tiempos en los desplazamientos de las mujeres ocupadas. 
Figura 4

TIEMPOS MEDIOS DE LOS DESPLAZAMIENTOS SEGÚN ACTIVIDAD Y GÉNERO (MINUTOS)

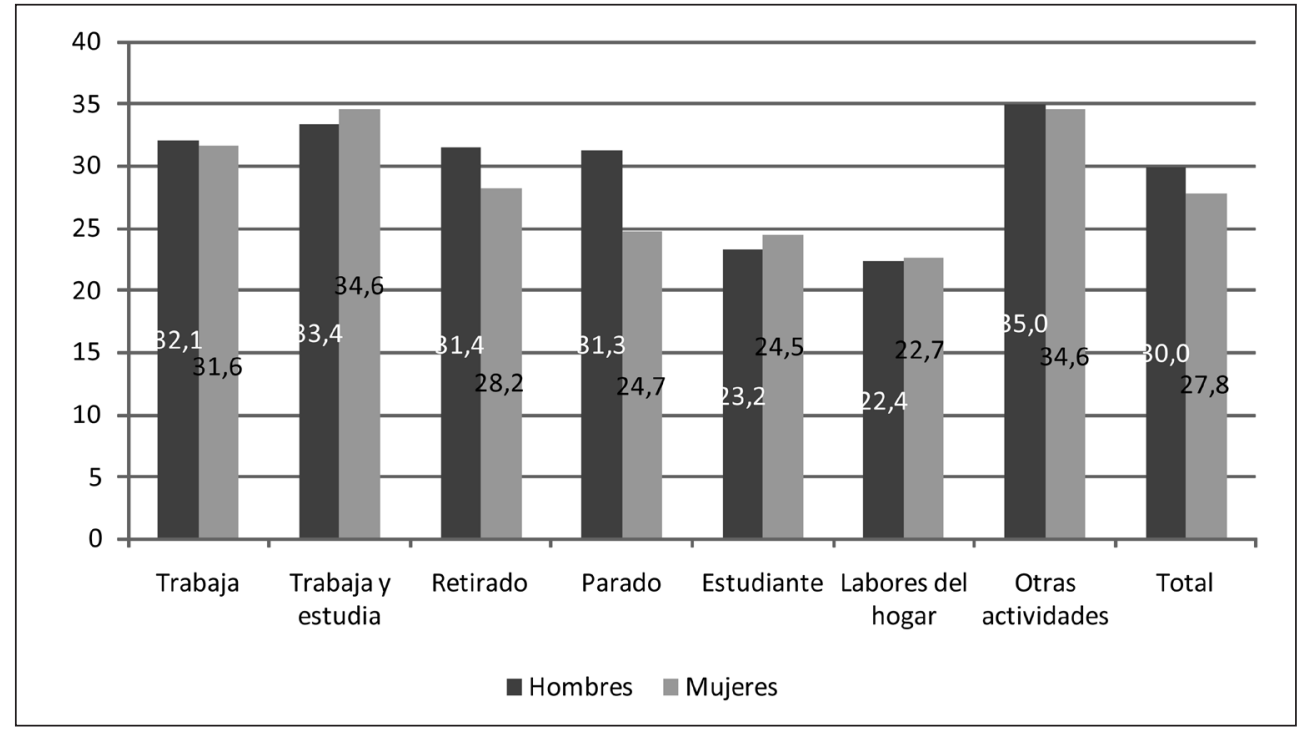

Fuente: Elaboración propia a partir de la EDM de 2004.

\section{Las diferencias de género se acentúan entre la población inmigrante}

Junto a la edad y la actividad, la raza y la etnia constituye una variable explicativa de especial importancia en la movilidad por género (Sabaté, Rodríguez y Díaz, 1995; Miralles y Cebollada, 2009). La creciente inmigración extranjera ha proporcionado un espectacular incremento de la población en la Comunidad de Madrid (Pozo, 2005), que se traduce en un crecimiento de la movilidad cotidiana e incide en el reparto modal, al aumentar la demanda de transporte público en relación con un colectivo muy dependiente de estos modos de transporte (García y Gutiérrez, 2007). Entre la población extranjera, en ambos sexos, se utiliza mucho más el transporte público que entre los españoles (Tabla 5). La proporción de desplazamientos en transporte público es mucho mayor entre las mujeres extranjeras que entre las españolas, pues las primeras suelen desplazarse más por motivos laborales, muchas veces para trabajar en el servicio doméstico y cuidado de personas mayores en espacios residenciales de rentas altas, alejados de sus lugares de residencia. Mientras, las españolas se desplazan más a pie y, sobre todo, usan más el coche (15 puntos porcentuales más que las extranjeras). Destaca como el uso del coche es mayor entre las mujeres españolas que entre los hombres extranjeros.

Efectivamente, aparecen diferencias en los motivos de los desplazamientos entre las mujeres españolas y extranjeras (Tabla 6). Mientras el $44 \%$ de las mujeres extranjeras se desplaza por motivos laborales, entre las españolas la proporción desciende al 28\%. La situación se invierte en el caso del ocio y deporte (4,3\% y $8,5 \%$ respectivamente), pero sobre todo en los desplazamientos por actividades relacionadas con el mantenimiento del hogar y sus 
miembros (compras de alimentos, prestaciones sanitarias, gestiones administrativas y otras tareas domésticas y de cuidado), donde en las mujeres españolas casi duplica la proporción de desplazamientos por estos motivos. En este sentido, destaca el desigual reparto del trabajo productivo y reproductivo entre hombres y mujeres según la nacionalidad, involucrándose más los hombres españoles por motivos sociales y culturales.

Tabla 5

MODO DE LOS DESPLAZAMIENTOS SEGÚN NACIONALIDAD Y GÉNERO, 2004 (PORCENTAJES)

\begin{tabular}{|c|l|c|c|c|c|}
\hline Género & \multicolumn{1}{|c|}{ Nacionalidad } & A pie & Transporte público & Coche & Otros modos \\
\hline \multirow{3}{*}{ Hombres } & Española & 25,3 & 27,4 & 43,1 & 4,2 \\
\cline { 2 - 6 } & Extranjera & 25,6 & 44,5 & 26,7 & 3,3 \\
\cline { 2 - 6 } & Total & 25,3 & 28,6 & 42,0 & 4,2 \\
\hline \multirow{3}{*}{ Mujeres } & Española & 36,5 & 33,0 & 28,2 & 2,4 \\
\cline { 2 - 6 } & Extranjera & 32,8 & 51,5 & 13,4 & 2,4 \\
\cline { 2 - 6 } & Total & 36,2 & 34,2 & 27,2 & 2,4 \\
\hline
\end{tabular}

Fuente: Elaboración propia a partir de la EDM de 2004.

Tabla 6

MOTIVO DE LOS DESPLAZAMIENTOS SEGÚN NACIONALIDAD Y GÉNERO, 2004 (PORCENTAJES)

\begin{tabular}{|l|l|c|c|c|c|c|}
\hline Género & Nacionalidad & Trabajo & Estudios & Compras & Ocio y deporte & $\begin{array}{c}\text { Otros motivos } \\
\text { reproductivos }\end{array}$ \\
\hline \multirow{2}{*}{ Hombres } & Española & 45,0 & 20,8 & 6,3 & 9,6 & 18,4 \\
\cline { 2 - 7 } & Extranjera & 57,9 & 20,9 & 3,6 & 4,8 & 12,9 \\
\cline { 2 - 7 } & Total & 45,8 & 20,8 & 6,1 & 9,3 & 18,0 \\
\hline \multirow{2}{*}{ Mujeres } & Española & 28,3 & 18,0 & 15,4 & 8,5 & 29,8 \\
\cline { 2 - 7 } & Extranjera & 44,0 & 17,1 & 10,7 & 4,3 & 23,9 \\
\cline { 2 - 7 } & Total & 29,4 & 17,9 & 15,1 & 8,2 & 29,4 \\
\hline
\end{tabular}

Fuente: Elaboración propia a partir de la EDM de 2004.

Las diferencias en el reparto modal y en los motivos de desplazamiento se traducen en tiempos medios en los desplazamientos superiores entre los extranjeros, tanto en las mujeres como los hombres (Figura 5). Además de la mayor utilización de transporte público, la población extranjera suele realizar desplazamientos más largos y complejos. Esto es significativo en aquellas que residen en las zonas del centro la ciudad o de los municipios suburbanos del sur o el este de la ciudad y trabajan en servicio doméstico en las zonas residenciales del oeste o el norte (Díaz, et al., 2009). 
Figura 5

TIEMPOS MEDIOS DE LOS DESPLAZAMIENTOS SEGÚN NACIONALIDAD Y GÉNERO (MINUTOS)

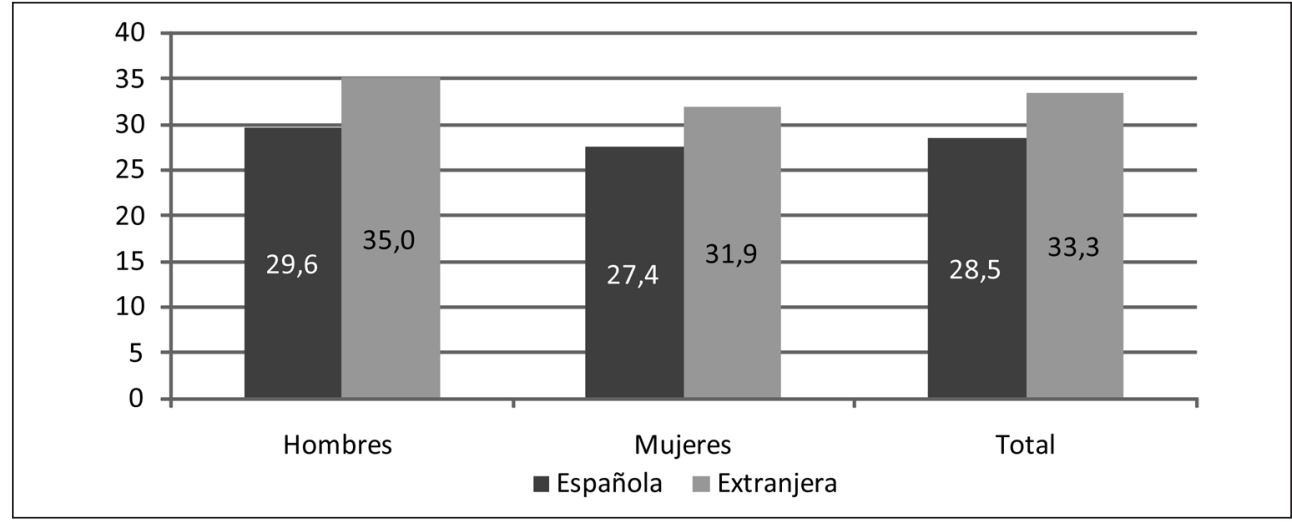

Fuente: Elaboración propia a partir de la EDM de 2004.

\section{LAS DIFERENCIAS EN LA MOVILIDAD DE GÉNERO SEGÚN LOS TIPOS DE ESPACIOS METROPOLITANOS}

Las diferencias en la movilidad según género y su caracterización sociodemográfica presentan diferencias territoriales, según el perfil de los espacios metropolitanos. En el actual modelo metropolitano interesa conocer cómo afecta a las diferencias de género la dependencia del coche que se produce en los espacios de expansión periurbana (las zonas más dinámicas del área metropolitana). Para ello hemos establecido una agrupación de los municipios de la Comunidad de Madrid según grandes ámbitos territoriales (ver apartado II), separando la ciudad central de Madrid, el espacio metropolitano tradicional, el oeste metropolitano, el espacio periurbano y los municipios rurales. En este apartado, nos centraremos además en los grupos de edades de 26 a 30 y de 31 a 40 años, donde como hemos visto la carga de trabajo reproductivo fuerza a las mujeres a diferenciar su movilidad. El análisis territorial descubre marcados contrastes en la movilidad y en sus diferencias de género.

\section{Diferencias territoriales y de género en la intensidad en la movilidad}

Los residentes en la ciudad de Madrid tienen una movilidad muy intensa, tanto entre los hombres como en las mujeres, realizando 2,7 desplazamientos diarios los dos grupos. Fuera de la ciudad de Madrid la movilidad se reduce y las diferencias de género aumentan. Esa reducción es especialmente llamativa en los espacios periurbanos, donde la necesidad de desplazamientos más largos y mecanizados para alcanzar las actividades reduce el número de desplazamientos tanto en hombres como en mujeres. Además, en el espacio periurbano aparecen diferencias entre hombres y mujeres. Los hombres se mueven menos (1,8 desplazamientos diarios por 2 desplazamientos diarios de las mujeres), concentrando su movilidad en desplazamientos por trabajo y estudio y reducen otros motivos de desplazamientos. Estas diferencias son similares en los grandes ámbitos del área metropolitana, de manera que entre 
hombres y mujeres de los municipios suburbanos tradicionales y del oeste aparece siempre una brecha de entorno a 0,2 desplazamientos, a pesar de las diferencias socioeconómicas y territoriales que existen entre los mismos (Tabla 7). La división sexual del trabajo y los roles de género explican las diferencias en la movilidad cotidiana por la complementariedad de actividades productivas y reproductivas de las mujeres de clase media.

Esa situación hay que matizarla cuando analizamos los grupos de edades de 26 a 40 años . Entre 26 y 30 años las diferencias de género son pequeñas, incluso en el oeste los hombres realizan más desplazamientos que las mujeres (probablemente por un acceso al coche en edades más jóvenes entre los hombres). Sin embargo, entre 31 y 40 años, las mujeres realizan muchos más desplazamientos que los hombres en todos los ámbitos. Las diferencias son ligeramente más altas en los grandes municipios metropolitanos (1,3 desplazamientos diarios más que los hombres) que en el oeste o en los espacios periurbanos $(1,1)$.

Tabla 7

RATIO DE DESPLAZAMIENTOS POR PERSONA SEGÚN GRANDES ÁMBITOS ESPACIALES

\begin{tabular}{|l|c|c|c|c|c|c|}
\hline \multirow{2}{*}{ Espacios } & \multicolumn{2}{|c|}{$\begin{array}{c}\text { Ratios (desplazamientos/ } \\
\text { persona) }\end{array}$} & \multicolumn{2}{c|}{ Población entre 26 y 30 años } & \multicolumn{2}{c|}{ Población entre 31 y 40 años } \\
\cline { 2 - 8 } & Hombres & Mujeres & Hombres & Mujeres & Hombres & Mujeres \\
\hline Madrid & 2,70 & 2,74 & 2,66 & 2,78 & 2,74 & 3,59 \\
\hline $\begin{array}{l}\text { Grandes municipios } \\
\text { metropolitanos }\end{array}$ & 2,11 & 2,36 & 1,79 & 2,08 & 2,06 & 3,36 \\
\hline Oeste metropolitano & 2,20 & 2,42 & 2,37 & 2,09 & 2,20 & 3,31 \\
\hline $\begin{array}{l}\text { Otros municipios } \\
\text { metropolitanos }\end{array}$ & 2,36 & 2,45 & 2,10 & 2,28 & 2,50 & 3,67 \\
\hline Periurbano & 1,85 & 2,04 & 1,59 & 1,98 & 1,95 & 3,05 \\
\hline Rurales & 0,68 & 0,75 & 0,52 & 0,65 & 0,71 & 1,24 \\
\hline Comunidad de Madrid & 2,31 & 2,46 & 2,15 & 2,33 & 2,35 & 3,35 \\
\hline
\end{tabular}

Fuente: Elaboración propia a partir de la EDM de 2004.

\section{Diferentes distribuciones según motivos de viaje}

Las diferencias en la intensidad de la movilidad se relacionan con una diferente distribución de desplazamientos según motivos. En el espacio denso del municipio de Madrid, donde las oportunidades son mayores, la movilidad de mujeres y hombres es muy diversa según motivos, de manera que la proporción de desplazamientos al trabajo o a estudios es más reducida en ambos sexo, mientras aumentan los desplazamientos a otras actividades (Tabla 8). Por el contrario, en el área metropolitana crecen los desplazamientos por trabajo o estudio entre los hombres, superando en todos los ámbitos el 70\% (ligeramente superior en el oeste, donde las tasas de actividad son más altas), mientras en las mujeres solo en el oeste se supera ligeramente el $50 \%$ y es todavía menor en los municipios metropolitanos tradicionales y los periurbanos. 
Es de destacar las proporciones de desplazamientos a compras, con dos situaciones claramente diferenciadas entre los municipios metropolitanos tradicionales, por un lado, y el oeste y el espacio periurbano por otro. En los primeros, la edad media es más alta, las tasas de ocupación más bajas y la proporción de extranjeras mayor, a lo que se une un espacio denso donde las oportunidades para los desplazamientos cortos a compras son mayores. Eso explica que entre las mujeres la proporción de desplazamientos a compras en estos espacios sea muy superior a los espacios del oeste y sobre todo del periurbano. Es en estos dos últimos ámbitos, donde se ha consolidado el modelo de consumo vinculado a grandes centros comerciales (compra semanal y en coche), que reduce los desplazamientos diarios femeninos e involucra más al hombre en estas tareas. A la vez, en el oeste y el periurbano los desplazamientos a otras tareas reproductivas tienen más peso entre las mujeres. Estos desplazamientos vinculados al ámbito doméstico y reproductivo no pueden suprimirse y son realizados fundamentalmente por las mujeres.

La clase social y la nacionalidad son determinantes en las mayores desigualdades de género en la distribución de los desplazamientos según motivos, que se alcanzan entre las mujeres en las edades donde se incrementa el trabajo reproductivo, pero además en aquellas que residen en los municipios periurbanos (Tabla 9). Las proporciones de desplazamientos al trabajo y estudios en hombres y mujeres entre 26 y 30 años son muy elevadas en ambos géneros, aunque más elevadas en Madrid y sobre todo en el oeste (donde las rentas y las tasas de actividad son más altas). No obstante, en todos los casos, también en el oeste, existe una brecha de género de alrededor de 14 puntos porcentuales.

Pero lo más interesante es como la mayor caída del porcentaje de movilidad al trabajo se da en el grupo de mujeres de 31 a 40 años residentes en el espacio periurbano. Esto se relaciona con el peso que cobran los desplazamientos por otras tareas reproductivas, que llegan a suponer casi un 52\% de los movimientos de estas mujeres, con una brecha de género muy alta. En estos espacios determinadas actividades relacionadas con el hogar (cuidado de los miembros, médico, trámites burocráticos, etc.) son más incompatibles con la localización y los horarios laborales y son asumidos por las mujeres.

Tabla 8

DESPLAZAMIENTOS SEGÚN MOTIVOS Y GRANDES ÁMBITOS METROPOLITANOS (PORCENTAJES)

\begin{tabular}{|l|c|c|c|c|c|c|c|c|}
\hline \multirow{2}{*}{ Espacios } & \multicolumn{2}{|c|}{ Trabajo y estudios } & \multicolumn{2}{c|}{ Compras } & \multicolumn{2}{c|}{ Ocio } & \multicolumn{2}{c|}{$\begin{array}{c}\text { Otros motivos } \\
\text { reproductivos }\end{array}$} \\
\cline { 2 - 10 } & Hombres & Mujeres & Hombres & Mujeres & Hombres & Mujeres & Hombres & Mujeres \\
\hline Madrid & 65,0 & 48,0 & 6,6 & 14,8 & 3,7 & 6,5 & 24,7 & 30,7 \\
\hline $\begin{array}{l}\text { Grandes municipios } \\
\text { metropolitanos }\end{array}$ & 70,0 & 46,9 & 5,8 & 17,2 & 2,6 & 4,5 & 21,5 & 31,3 \\
\hline Oeste metropolitano & 71,7 & 50,5 & 4,5 & 12,6 & 2,2 & 3,7 & 21,6 & 33,3 \\
\hline $\begin{array}{l}\text { Otros municipios } \\
\text { metropolitanos }\end{array}$ & 70,4 & 45,9 & 4,8 & 14,8 & 2,3 & 4,0 & 22,4 & 35,3 \\
\hline Periurbano & 69,5 & 48,6 & 4,6 & 11,9 & 2,0 & 4,3 & 23,9 & 35,2 \\
\hline Rurales & 67,9 & 45,1 & 7,3 & 15,6 & 2,6 & 4,0 & 22,2 & 35,4 \\
\hline
\end{tabular}

Fuente: Elaboración propia a partir de la EDM de 2004. 
Tabla 9

DESPLAZAMIENTOS SEGÚN MOTIVOS, EDADES Y GRANDES ÁMBITOS METROPOLITANOS (PORCENTAJES)

\begin{tabular}{|c|c|c|c|c|c|c|c|c|}
\hline \multicolumn{9}{|c|}{ Población entre 26 y 30 años } \\
\hline \multirow{2}{*}{ Espacios } & \multicolumn{2}{|c|}{ Trabajo y estudios } & \multicolumn{2}{|c|}{ Compras } & \multicolumn{2}{|c|}{ Ocio } & \multicolumn{2}{|c|}{$\begin{array}{l}\text { Otros motivos } \\
\text { reproductivos }\end{array}$} \\
\hline & Hombres & Mujeres & Hombres & Mujeres & Hombres & Mujeres & Hombres & Mujeres \\
\hline Madrid & 76,1 & 67,7 & 3,5 & 7,0 & 1,1 & 2,2 & 19,3 & 23,1 \\
\hline $\begin{array}{l}\text { Grandes municipios } \\
\text { metropolitanos }\end{array}$ & 76,3 & 63,7 & 3,6 & 10,7 & 1,2 & 2,2 & 18,9 & 23,5 \\
\hline Oeste metropolitano & 82,1 & 68,1 & 1,5 & 6,3 & 2,1 & 1,3 & 14,3 & 24,4 \\
\hline $\begin{array}{l}\text { Otros municipios } \\
\text { metropolitanos }\end{array}$ & 76,6 & 62,8 & 5,2 & 15,0 & 0,8 & 2,7 & 17,4 & 19,4 \\
\hline Periurbano & 77,9 & 64,0 & 2,9 & 6,1 & 0,9 & 1,3 & 18,4 & 28,6 \\
\hline Rurales & 71,0 & 60,7 & 5,7 & 12,7 & 1,4 & 2,4 & 21,9 & 24,3 \\
\hline \multicolumn{9}{|c|}{ Población entre 31 y 40 años } \\
\hline Madrid & 71,0 & 48,8 & 4,2 & 9,4 & 2,0 & 4,4 & 22,7 & 37,4 \\
\hline $\begin{array}{l}\text { Grandes municipios } \\
\text { metropolitanos }\end{array}$ & 70,4 & 34,3 & 6,1 & 13,2 & 2,1 & 4,1 & 21,5 & 48,4 \\
\hline Oeste metropolitano & 73,8 & 38,1 & 4,4 & 11,9 & 1,3 & 2,9 & 20,5 & 47,1 \\
\hline $\begin{array}{l}\text { Otros municipios } \\
\text { metropolitanos }\end{array}$ & 76,3 & 29,8 & 3,9 & 12,6 & 0,8 & 3,7 & 19,0 & 53,9 \\
\hline Periurbano & 73,4 & 34,0 & 4,3 & 10,5 & 1,9 & 3,6 & 20,5 & 51,9 \\
\hline Rurales & 73,4 & 32,6 & 7,2 & 12,7 & 1,7 & 2,1 & 17,7 & 52,6 \\
\hline
\end{tabular}

Fuente: Elaboración propia a partir de la EDM de 2004.

\section{Las diferencias espaciales en el reparto modal}

Las mayores diferencias de género en la movilidad según ámbitos territoriales se dan en el reparto según modos de transporte (Tabla 10). En la ciudad de Madrid y, sobre todo, en los grandes y densos municipios metropolitanos tradicionales la proporción de desplazamientos andando es muy importante, especialmente entre las mujeres. Estos desplazamientos andando se reducen significativamente en el oeste y en el periurbano, aunque la brecha de género es mucho menor en el oeste (lo que se explica por el mayor peso de las tareas reproductivas en el periurbano y su menor poder adquisitivo).

Las diferencias entre ámbitos son también llamativas en el uso del transporte público. En los municipios densos las mujeres hacen un uso mucho mayor transporte público, es el caso de Madrid y, en menor medida, en los municipios metropolitanos tradicionales. Sin embargo, en el periurbano y en el oeste, con modelos dispersos y de baja densidad donde el transporte público sirve peor las necesidades de los desplazamientos (en especial los más cortos), el uso 
del transporte público se reduce, y lo hace sobre todo entre las mujeres, desapareciendo la diferencia con los hombres.

Así, en el oeste y los municipios periurbanos se intensifica el uso del coche. La proporción de desplazamientos en coche crece tanto en hombres como en mujeres, con valores muy altos en el oeste en los dos grupos y una brecha muy baja $(2,2 \%)$, y valores elevados en el periurbano, aunque sin alcanzar los del oeste en el caso de las mujeres, con una brecha de género de casi 12 puntos porcentuales. No obstante, este valor es muy inferior al de los municipios suburbanos, que reducen todavía más el uso del coche entre las mujeres respecto a los hombres.

Es interesante analizar el diferente uso del coche que se hace entre ámbitos en los grupos de 26 a 40 años (Tabla 11). En los municipios metropolitanos tradicionales, la combinación de trabajo productivo y reproductivo se traduce en un incremento de los desplazamientos a pie en las mujeres de 31 a 40 años, con un aumento de la brecha de género de 10 a 31 puntos porcentuales, y un fuerte descenso de la movilidad mecanizada (las diferencias en el uso del transporte público se acortan y se incrementan especialmente en el uso del coche). En el oeste crece la movilidad peatonal, pero con un peso mucho menor y menores diferencias de género. Esto no ocurre en el periurbano, donde la diferencia de género en la movilidad peatonal se mantiene.

Pero lo más llamativo es que tanto en el oeste como en el periurbano crece la movilidad mecanizada, en especial la relacionada con el uso del transporte privado, de manera que en los dos ámbitos se reducen las diferencias de género en el uso del coche entre el grupo de edad de 31 a 40 años. La necesidad del coche en estos espacios, todavía mayor en el caso de los desplazamientos por trabajo reproductivo, donde hay que encadenar diferentes etapas, conduce a las mujeres a un uso mucho mayor del coche. Además, las mayores tasas de motorización y en consecuencia el mayor acceso de las mujeres al coche, en especial en el oeste, parece confirmar la afirmación de Best y Lanzendorf (2005), que concluyen que mujer y hombre utilizan el coche en igual proporción cuando tienen plena disponibilidad de mismo.

Tabla 10

DISTRIBUCIÓN MODAL SEGÚN GRUPOS DE EDAD Y TIPOS DE ESPACIOS (PORCENTAJES)

\begin{tabular}{|l|c|c|c|c|c|c|c|c|}
\hline & \multicolumn{2}{|c|}{ A pie } & \multicolumn{2}{c|}{ Transporte Público } & \multicolumn{2}{c|}{ Transporte privado } & \multicolumn{2}{c|}{ Otros modos } \\
\hline & Hombres & Mujeres & Hombres & Mujeres & Hombres & Mujeres & Hombres & Mujeres \\
\hline Madrid & 24,6 & 33,1 & 37,0 & 44,2 & 34,5 & 20,3 & 3,9 & 2,3 \\
\hline $\begin{array}{l}\text { Grandes municipios } \\
\text { metropolitanos }\end{array}$ & 30,7 & 47,6 & 19,7 & 23,1 & 45,9 & 27,4 & 3,7 & 1,9 \\
\hline Oeste & 14,2 & 17,9 & 18,4 & 19,1 & 62,0 & 59,8 & 5,5 & 3,1 \\
\hline $\begin{array}{l}\text { Otros municipios } \\
\text { metropolitanos }\end{array}$ & 27,0 & 42,9 & 12,6 & 15,2 & 55,6 & 40,4 & 4,8 & 1,6 \\
\hline Periurbano & 19,9 & 32,9 & 12,9 & 14,1 & 61,7 & 49,9 & 5,5 & 3,1 \\
\hline Rurales & 26,1 & 38,7 & 8,2 & 11,4 & 57,3 & 43,3 & 8,4 & 6,6 \\
\hline
\end{tabular}

Fuente: Elaboración propia a partir de la EDM de 2004. 
Tabla 11

DISTRIBUCIÓN MODAL SEGÚN GRUPOS DE EDAD Y TIPOS DE ESPACIOS (PORCENTAJES)

\begin{tabular}{|c|c|c|c|c|c|c|c|c|c|}
\hline \multicolumn{10}{|c|}{ Población entre 26 y 30 años } \\
\hline & \multicolumn{3}{|c|}{ Grandes municipios metropolitanos } & \multicolumn{3}{|c|}{ Oeste } & \multicolumn{3}{|c|}{ Periurbano } \\
\hline & Hombres & Mujeres & Brecha & Hombres & Mujeres & Brecha & Hombres & Mujeres & Brecha \\
\hline A pie & 15 & 25,6 & 10,6 & 3,9 & 8,2 & 4,3 & 11,1 & 25,2 & 14,1 \\
\hline Público & 24,7 & 32,1 & 7,4 & 22,9 & 27,6 & 4,7 & 15,4 & 22,3 & 6,9 \\
\hline Privado & 57,5 & 40,9 & $-16,6$ & 69,8 & 62,8 & -7 & 71 & 52,3 & $-18,7$ \\
\hline Otros & 2,9 & 1,3 & $-1,6$ & 3,4 & 1,4 & -2 & 2,4 & 0,3 & $-2,1$ \\
\hline \multicolumn{10}{|c|}{ Población entre 31 y 40 años } \\
\hline & \multicolumn{3}{|c|}{ Grandes municipios metropolitanos } & \multicolumn{3}{|c|}{ Oeste } & \multicolumn{3}{|c|}{ Periurbano } \\
\hline & Hombres & Mujeres & Brecha & Hombres & Mujeres & Brecha & Hombres & Mujeres & Brecha \\
\hline Apie & 12,8 & 44 & 31,2 & 6 & 17,8 & 11,8 & 12,4 & 26,9 & 14,5 \\
\hline Público & 16 & 17,8 & 1,8 & 20,9 & 12,8 & $-8,1$ & 13,2 & 8,7 & $-4,5$ \\
\hline Privado & 67,7 & 37,2 & $-30,5$ & 70,3 & 68,8 & $-1,5$ & 72,5 & 63,8 & $-8,7$ \\
\hline Otros & 3,6 & 1 & $-2,6$ & 2,7 & 0,7 & -2 & 1,9 & 0,6 & $-1,3$ \\
\hline
\end{tabular}

Fuente: Elaboración propia a partir de la EDM de 2004.

Uno de los cambios a los que se hace referencia en las áreas metropolitanas es el que se refiere a las formas de consumo y su vinculación a las grandes superficies comerciales. Como hemos visto, tanto en el oeste como en los espacios periurbanos se reduce la proporción de desplazamientos a compras. Se pasa de un modelo de compra diario en el barrio a una compra semanal en el centro comercial. Esto en principio se relaciona con un mayor uso del coche, acortándose la brecha de género en un motivo de viaje tradicionalmente vinculado con las mujeres y desplazamientos peatonales. Efectivamente, si nos fijamos en el reparto modal de los desplazamientos a compras según ámbitos metropolitanos esto es así en los municipios del oeste. En estos municipios las mujeres se desplazan a pie tan sólo en el $31 \%$ de los casos, mientras usan el coche en el $61 \%$ de los desplazamientos a compras, con una brecha de género muy baja. Pero en el espacio periurbano la importancia de los desplazamientos a pie se mantiene entre las mujeres, con un porcentaje todavía elevado (57\%), mientras que a pesar del incremento de los desplazamientos en coche, la brecha de género en este modo es muy alta (-25\%). Por el contrario, el modelo de consumo tradicional todavía se mantiene en los grandes municipios suburbanos, donde casi el $70 \%$ de los desplazamientos a compras femeninos son a pie y apenas usan el coche (Figura 6). 
Figura 6

REPARTO MODAL DE LOS DESPLAZAMIENTOS A COMPRAS Y TIPOS DE ESPACIOS (PORCENTAJES)

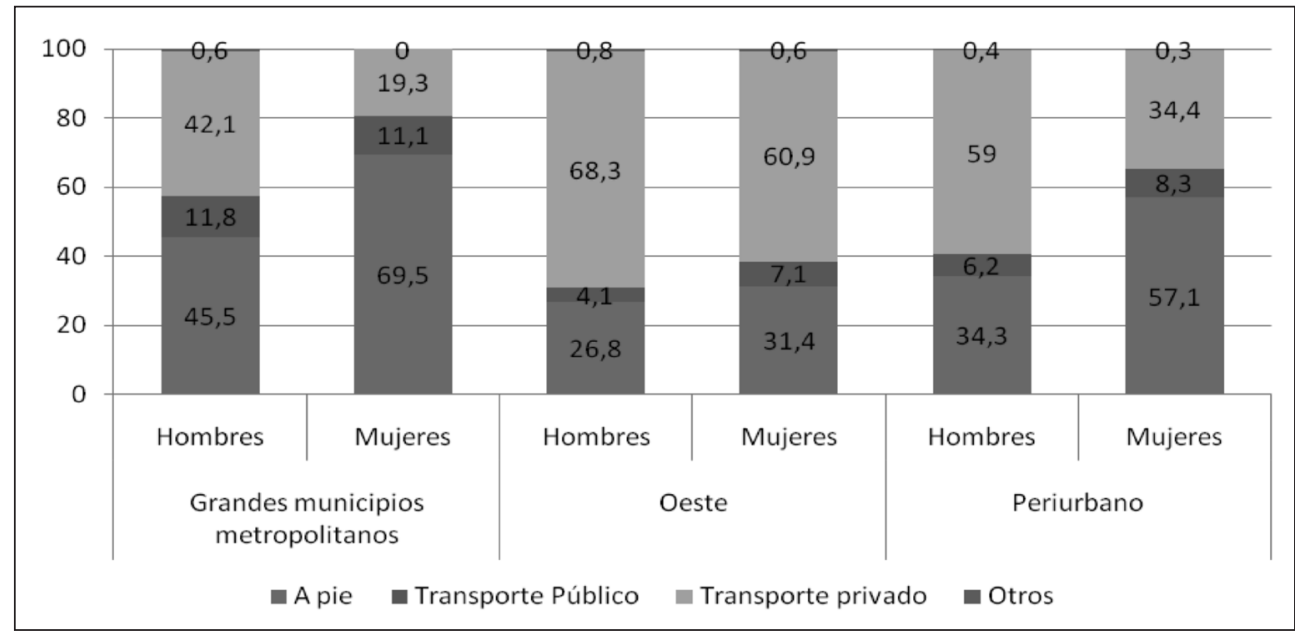

Fuente: Elaboración propia a partir de la EDM de 2004.

\section{Los tiempos dedicados a los desplazamientos}

Como ya hemos visto, en la Comunidad de Madrid las mujeres emplean menos tiempo en los desplazamientos que los hombres. Ese menor tiempo lo encontramos en todos los ámbitos espaciales, aunque con diferencias llamativas entre ellos (Tabla 12). El modelo urbano del municipio de Madrid favorece los desplazamientos en transporte público pero con distancias relativamente altas, lo que explica que sea en este ámbito donde los tiempos empleados en los desplazamientos tengan los valores más elevados, tanto entre los hombres como entre las mujeres. Sin embargo, las menores diferencias de género en el reparto modal hacen que sea también en el interior de Madrid donde la brecha de género es menor, aumentando en los municipios metropolitanos tradicionales, junto con los municipios del periurbano y los municipios del oeste.

Tabla 12

TIEMPOS DE DESPLAZAMIENTO SEGÚN GÉNERO Y TIPOS DE ESPACIOS (MINUTOS)

\begin{tabular}{|l|c|c|c|c|}
\hline & Hombres & Mujeres & Brecha & Brecha (\%) \\
\hline Madrid & 31,1 & 29,9 & $-1,2$ & $-4,0$ \\
\hline Grandes municipios metropolitanos & 27,6 & 23,8 & $-3,9$ & $-13,9$ \\
\hline Oeste & 30,0 & 25,3 & $-4,6$ & $-15,4$ \\
\hline Otros municipios metropolitanos & 26,9 & 21,2 & $-5,7$ & $-21,1$ \\
\hline Periurbano & 27,4 & 23,6 & $-3,8$ & $-14,0$ \\
\hline Rurales & 27,9 & 24,0 & $-3,9$ & $-14,0$ \\
\hline
\end{tabular}

Fuente: Elaboración propia a partir de la EDM de 2004. 
La distribución de los tiempos medios de los desplazamientos según motivos también presenta diferencias espaciales y de género (Tabla 13). Es significativo que en la capital las mujeres emplean más tiempo en desplazarse que los hombres para ir al trabajo, al lugar de estudios o de ocio. La mayor oferta de transporte público y su mayor utilización por las mujeres explica esta situación. En el resto de los ámbitos, las mujeres emplean menos tiempo que los hombres en los desplazamientos al trabajo, bien porque tienden a buscar empleos cerca del hogar para poder compatibilizar los dos tipos de trabajo, bien porque se ven obligadas a utilizar el transporte privado por la escasez de transporte público. En los desplazamientos a compras y ocio, los tiempos son siempre inferiores en el caso de las mujeres, salvo en los desplazamientos a ocio en el periurbano. Además, cabe destacar los mayores tiempos a compras en el municipio de Madrid y el aumento considerable del tiempo empleado en los desplazamientos por ocio en las zonas rurales, consecuencia de la menor oferta, lo que les obliga a desplazarse a mayores distancias. Pero lo más importante es que las mayores diferencias de género se producen en el caso de las tareas reproductivas, y en especial en los municipios del oeste y, sobre todo, del periurbano, donde las mujeres dedican hasta 6 minutos menos que los hombres a este tipo de desplazamientos. Esto se explica por la mayor dependencia del coche y la realización de los desplazamientos más largos por parte de los hombres.

Tabla 13

TIEMPOS DE DESPLAZAMIENTO SEGÚN MOTIVO, GÉNERO Y TIPOS DE ESPACIOS (MINUTOS)

\begin{tabular}{|l|r|r|r|r|r|r|r|r|}
\hline & \multicolumn{2}{|c|}{ Trabajo y estudios } & \multicolumn{2}{|c|}{ Compras } & \multicolumn{2}{c|}{ Ocio } & \multicolumn{2}{c|}{$\begin{array}{l}\text { Otros motivos } \\
\text { reproductivos }\end{array}$} \\
\hline & Hombres & Mujeres & Hombres & Mujeres & Hombres & Mujeres & Hombres & Mujeres \\
\hline Madrid & 33,2 & 34,5 & 22,6 & 21,6 & 32,7 & 33,2 & 27,5 & 25,8 \\
\hline $\begin{array}{l}\text { Grandes municipios } \\
\text { metropolitanos }\end{array}$ & 30,3 & 29,7 & 18,7 & 16,4 & 25,0 & 25,4 & 21,7 & 18,7 \\
\hline Oeste & 32,9 & 32,1 & 17,7 & 16,5 & 30,8 & 28,2 & 22,5 & 18,0 \\
\hline $\begin{array}{l}\text { Otros municipios } \\
\text { metropolitanos }\end{array}$ & 29,5 & 26,5 & 16,9 & 15,0 & 31,2 & 22,8 & 20,3 & 16,7 \\
\hline Periurbano & 29,4 & 29,0 & 16,8 & 16,1 & 34,4 & 36,7 & 23,2 & 17,1 \\
\hline Rurales & 30,1 & 28,7 & 18,1 & 14,5 & 49,6 & 48,9 & 21,8 & 19,3 \\
\hline
\end{tabular}

Fuente: Elaboración propia a partir de la EDM de 2004.

El modo de desplazamiento es determinante en los tiempos finales dedicados a los mismos (Tabla 14). El modo de transporte que más tiempo consume, tanto en hombres como en mujeres, es el transporte público. Además, los tiempos en transporte público aumentan al alejarnos del municipio de Madrid, pero en cualquier caso las diferencias entre sexos no son altas. Si lo son, en cambio, en los desplazamientos en coche, donde las mujeres tienen tiempos hasta un $20 \%$ inferiores a los de los hombres tanto en el oeste metropolitano como en los municipios periurbanos. En el municipio de Madrid los desplazamiento en coche emplean 
más tiempo, consecuencia de la mayor congestión, pero las diferencias de género son mucho menores.

Como es lógico, los desplazamientos más cortos son los peatonales, que son más largos en Madrid, donde las actividades disponibles en desplazamientos cortos son mucho mayores, y descienden a medida que nos alejamos de la capital. Pero lo destacable es como nuevamente los tiempos empleados por las mujeres en estos desplazamientos andando son en todos los ámbitos alrededor de un $10 \%$ menores que en los hombres.

Tabla 14

TIEMPOS DE DESPLAZAMIENTOS SEGÚN MODOS DE TRANSPORTE (MINUTOS)

\begin{tabular}{|l|c|c|c|c|c|c|c|c|}
\hline & \multicolumn{2}{|c|}{ A pie } & \multicolumn{2}{c|}{ Transporte Público } & Transporte privado & \multicolumn{2}{c|}{ Otros modos } \\
\hline & Hombres & Mujeres & Hombres & Mujeres & Hombres & Mujeres & Hombres & Mujeres \\
\hline Madrid & 18,2 & 16,1 & 42,8 & 42,6 & 27,9 & 24,7 & 29,5 & 29,4 \\
\hline $\begin{array}{l}\text { Grandes municipios } \\
\text { metropolitanos }\end{array}$ & 15,9 & 14,4 & 48,4 & 46,0 & 26,0 & 20,9 & 34,5 & 30,6 \\
\hline Oeste & 15,3 & 14,2 & 55,0 & 51,3 & 25,8 & 20,0 & 31,5 & 32,1 \\
\hline $\begin{array}{l}\text { Otros municipios } \\
\text { metropolitanos }\end{array}$ & 16,2 & 13,8 & 59,2 & 50,4 & 24,1 & 17,5 & 34,0 & 34,7 \\
\hline Periurbano & 14,4 & 12,2 & 62,2 & 63,5 & 24,5 & 19,5 & 25,9 & 28,5 \\
\hline Rurales & 14,4 & 12,4 & 73,9 & 71,7 & 27,4 & 20,6 & 28,4 & 31,9 \\
\hline
\end{tabular}

Fuente: Elaboración propia a partir de la EDM de 2004.

\section{CONCLUSIONES}

La vida cotidiana de hombres y mujeres es diferente. La de las mujeres es más compleja, ya que se responsabiliza del trabajo productivo y reproductivo, lo que se traduce en una movilidad cotidiana diferenciada. Como señalan Jaekel y van Geldermalsen (2006) las mujeres necesitan espacios urbanos multifuncionales para lograr el equilibrio de sus realidades cotidianas multitarea.

En este trabajo ha quedado demostrada la existencia de diferencias en los patrones de movilidad diaria entre hombres y mujeres en la Comunidad de Madrid. Como hemos visto, este diferente comportamiento se explica por diversos componentes, como las dinámicas demográficas, sociales, económicas y territoriales. El análisis de movilidad cotidiana según género nos ha permitido comprobar que:

- La diferente movilidad cotidiana entre hombres y mujeres sólo puede comprenderse por la complementariedad de las actividades productivas y reproductivas de las mujeres. Las mujeres, en su papel de amas de casa y adscritas al trabajo doméstico, ven restringido su ámbito espacial de la vida cotidiana a la esfera del barrio de residencia, realizan más desplazamientos, pero más cortos y se siguen desplazando mayoritariamente a pie y en transporte público, en especial en los ámbitos territoriales más densos y compactos del modelo metropolitano tradicional, si bien, su horizonte se amplía 
en el caso de las mujeres que han accedido al mercado de trabajo. Por el contrario, la movilidad de los hombres aparece espacialmente más amplia y condicionada no tanto por la proximidad del hogar, sino por las oportunidades laborales disponibles, lo que le convierte en el mayor usuario del transporte privado. Al igual que ha encontrado Hanson (2010), en Madrid las mujeres utilizan medios de transporte más sostenibles, tanto porque sus menores rentas no les permiten tener acceso al transporte privado en la misma proporción que los hombres, como por ideología. En definitiva, el género puede ser un agente del cambio en las conductas pro-ambientales.

- La edad adulta y la carga de los trabajos reproductivos en las mujeres constituye una característica fundamental que agrava el carácter comparativamente más reducido de la movilidad femenina.

- Las diferencias de género deben además analizarse en función de otras variables sociodemográficas, pues varían con fuerza en función de la nacionalidad o la actividad. La población extranjera, en ambos géneros, utiliza más el transporte público que la española. Además, las mujeres extranjeras realizan menos desplazamientos a pie que las mujeres españolas.

- La actividad es otra variable que afecta muy significativamente a los desplazamientos según género. Las mujeres ocupadas utilizan fundamentalmente el transporte público para ir al trabajo remunerado, mientras que los hombres utilizan bastante más el vehículo privado. En el caso de las mujeres que trabajan en las labores del hogar, los desplazamientos los realizan a pie, por tanto su movilidad es reducida y el barrio de residencia constituye el espacio de referencia en el funcionamiento cotidiano del hogar.

En este artículo hemos puesto especial atención en las diferencias de género en la movilidad según ámbitos territoriales. Se ha tratado de ver cómo inciden los modelos territoriales, en especial los antagónicos modelos de suburbanización concentrada de los municipios metropolitanos tradicionales y los nuevos desarrollos dispersos de los espacios de baja densidad del oeste y las zonas periurbanas. Se ha podido constatar que el nuevo modelo disperso obliga a realizar desplazamientos más largos y acrecienta las dificultades de las mujeres, en especial entre aquellas que tienen más problemas para acceder al coche. Las diferencias de género en la movilidad en estos espacios son significativas, todavía más en los municipios periurbanos, donde los niveles de renta más bajos y las menores tasas de motorización acrecientan esa diferente movilidad. Así, hemos encontrado como:

- Las mujeres de los municipios periurbanos de la Comunidad de Madrid realizan más desplazamientos que los hombres, sobre todo en el grupo de edad de 31 a 40 años, pero reducen significativamente el número de desplazamientos que realizan respecto a otros ámbitos metropolitanos como el oeste y sobre todo los municipios metropolitanos tradicionales.

- A la vez, la mayor caída de los desplazamientos al trabajo en las mujeres de 31 a 40 años se da también en los municipios periurbanos, donde el espacio disperso aleja la posibilidad compatibilizar empleo y trabajo reproductivo.

- Finalmente, se ha visto como la movilidad en estos espacios es muy dependiente del transporte privado, pero son los hombres los que hacen un uso intensivo del mismo y en desplazamientos más largos, mientras las mujeres lo usan menos y en desplazamientos más cortos. 
En resumen, los cambios que se están produciendo en el modelo metropolitano y los retos de futuro que suponen, la planificación conjunta de territorio, sistema de transporte y movilidad debe de tener en cuenta una visión más integral, en la que se incorpore la perspectiva de género en sus fases de diseño, proceso y ejecución. El conocimiento de las pautas de movilidad cotidiana de los hombres y las mujeres resulta fundamental para poder llevar a cabo actuaciones eficaces en la planificación del transporte en las áreas metropolitanas.

\section{BIBLIOGRAFÍA}

BEST, H. y LANZENDORF, M. (2005): «Division of labour and differences in metropolitan car use: An empirical study in Colgne, Germany». Journal of Transport Geography, 13 2, 109-121

CASADO, J. M. (2000) : Diferencias de género en los desplazamientos cotidianos por razones laborales. Instituto Valenciano de Investigaciones Económicas.

LA CARTA EUROPEA DE LA MUJER EN LA CIUDAD (1995). En Ciudades para un futuro más sostenible. Buenas prácticas del concurso Habitat II, Bruselas. Disponible en http://habitat.aq.upm.es/hpn/bp216.html.

COSTRAS, J. (1997): «La mobilité quotidienne et les inégalités de sexe à travers le prime des stadistiques». Recherches Féministes., 10, 77-90.

CEBOLLADA, A. Y MIRALLES, C. (2003): «Hábitos y percepciones de la movilidad cotidiana de las personas sin permiso de conducir». En J. Seguí (edit): Los servicios, los transportes y las redes territoriales. Els serveis, els transports i les xarxes territorials, Universitat de les Illes Balears; Asociación de Geógrafos Españoles (AGE), Grupo de Geografía de los Servicios, Palma de Mallorca.

CLOS, I. (1986) : «El viatge al treball a Barcelona i entorn». Documents d'Anàlisi Geogràfica, 8-9, 25-38.

CRISTALDI, F. (2005): «Commuting and gender in Italy: a methodological issue», The Professional Geographer, (2), 268-284.

DÍAZ, M. A. (1988): «Actividad, género y desplazamientos urbanos en Alcalá de Henares», en: Aportación española al XXVI Congreso Geográfico Internacional, Madrid, Real Sociedad Geográfica, 107-117.

DÍAZ, M. A. (1995): «El espacio-tiempo cotidiano», en: Ciudad y mujer. Nuevas visiones del espacio público y privado. Madrid, Seminario Permanente Ciudad y Mujer, Junta C.M. y Colegio Oficial de Arquitectos de Castilla-La Mancha, 107-117.

DÍAZ, M. A. Y JIMÉNEZ, F. J. (2003): «Transportes y movilidad: ¿Necesidades diferenciales según género?», Segundo Seminario Internacional sobre Género y Urbanismo. Infraestructuras para la vida cotidiana. ETSAM, UPM. Disponible en http://www.generourban.org/archivos/seminario_2002.htm (consulta 02-02-2007)

DÍAZ, M. A.; RODRÍGUEZ, J. M. Y GARCÍA, J. C. (2009): «Desplazamientos laborales y género en la Comunidad de Madrid a partir del censo de población 2001», En PONS, J.J et al. (Editores). Territorio y movilidad interior de la Población en España. Ed. EUNSA. Ediciones Universidad de Navarra. 235-242

DUPUY, G. (1995): «Les territories de l'automobile». Anthropos-Economica. París. 
FAGNANI, J. (1977): «Áctivités féminines et transports urbains». Annales de Géographie, 477, 543-561.

FAGNANI, J. (1986): «La durèe des trajets quotidiens: un enjeu pour les méres actives». Economie et Stadistiques, 185, 47-55.

GARCÍA, J. Y GUTIÉRREZ, J. (2007): «Pautas de la movilidad en la Comunidad de Madrid», Cuadernos de Geografía de la Universidad de Valencia, 81/82, 7-29.

GARCÍA, J.C. Y RODRÍGUEZ, J. (2008): «Movilidad cotidiana y género en la Comunidad de Madrid». XI Congreso Ibérico de Geografía. Alcalá de Henares.

GUTIÉRREZ, J. Y GARCÍA, J.C. (2007a): «La ciudad dispersa: cambios recientes en los espacios residenciales de la Comunidad de Madrid». Anales de Geografía de la Universidad Complutense, 27.1, 45-67.

GUTIÉRREZ, J. Y GARCÍA, J.C. (2007b): «New spatial patterns of mobility within the metropolitan area of Madrid: towards more complex and dispersed flow networks». Journal of Transport Geography, 15, 18-30.

HANSON, S. (2010): «Gender and mobility: new approaches for informing sustainability». Gender, Place \&Culture, 17, 5-23

HJORTHOL, R. J. (1998): Travel activities in everyday life: an analysis of women's and men's daily travel activities in Oslo. Dissertation for dr philos. Department of Sociology and Human Geography. University of Oslo, Oslo. Institute of Transport Economics, report 391.

HOWE Y O'CONNOR (1982): «Travel to work and labor participation of men and women in Australin Metropolitan Area», The Professional Geographer, 34, 50-64.

HOZ, D. de la (2008): «Factores sociales en las pautas de desplazamiento y uso del coche de la movilidad obligada de Madrid». Revista del Instituto de Estudios Económicos, 4, 53-68.

JAECKEL, M. y VAN GELDERMALSEN, M. (2006): «Equipment: daily infrastructures». En Urbanisme y génere: una visó necessària per a tothom. Diputación de Barcelona. Barcelona. http://www.urbanismeigenere.net/inside.asp?secao=doc_pon

LAW, R. (1999): «Beyond 'women and transport': towards new geographies of gender and daily mobility», Progress in Human Geography, 23 (4), 567-588.

LEE, B.S. Y MCDONALD, J.F. (2003): «Determinants of commuting time and distance for Seoul residents: the impact of family status on the commuting of women». Urban Studies, 40 (7). 1283-1302.

LEY, R. (1999): «Beyond 'women and transport': towards new geographies of gender and daily mobility», Progress in Human Geography, 23 (4), 567-588.

LITTLE, J. (1994): «Accessibility and transport», en Gender, Planning and the Policy Process, Pergamon Press, Oxford.

MADDENS, J. F. (1981): «Why women work closer to home?». Urban Studies, 18, 191-194

MELLA, J.M. Y LÓPEZ, A. (2006): «Forma urbana y movilidad sostenible: el caso de Madrid». Revista del Instituto Estudios Económicos, 1-2. 158-189.

MENDEZ, R. (2007): «Transformaciones económicas, entornos innovadores y reorganización de los territorios metropolitanos: algunas claves interpretativas». En Calatrava, A. y otros (coord..). Economías, mercados de trabajo y territorios metropolitanos en transformación. Ed. ARETHUSE. Madrid. 
MÉNDEZ, R. Y RODRÍGUEZ, J. (2007): «Transformaciones productivas y nuevas formas urbanas. Difusión de las actividades económicas en la región funcional de Madrid». Anales de Geografía de la Universidad Complutense de Madrid, 27.2, 105-134.

MIRALLES, C. (1998) «La movilidad de las mujeres en la ciudad. Un análisis desde la Ecología Urbana», Ecología Política, 15, 123-130.

MIRALLES, C. Y CEBOLLADA, A. (2009): «Movilidad cotidiana y sostenibilidad, una interpretación desde la Geografía Humana». Boletín de la Asociación de Geógrafos Españoles, 50, 193-216.

MONZÓN, A., VALDÉS, C. Y GENG XUE (2008): «Movilidad sostenible: una cuestión de lugar y género». Anuario de Movilidad, 2008, en publicación digital: http://www.racc. es/externos/fundacion/anuario2008esp/datos/media/racc_anuario_movilidad_2008.pdf.

POLK M., (2003): «Are women more potentially accommodating than men are to a sustainable transportation system in Sweden?» Transportation Research Part D, 8(2), 75-95

POLK M., (2004): «The influence of gender on daily car use and on willingness to reduce car use in Sweden». Journal of Transport Geography, 12, 185-195.

POZO, E. (2005): «Tendencias recientes en la evolución de la población de la comunidad de Madrid». Anales de Geografía de la Universidad Complutense, 25, 353-379.

RODRÍGUEZ, J.M. Y DÍAZ, M.A. (2007): «Género y participación laboral en la Comunidad de Madrid. Las dimensiones espaciales de un proceso de cambio». Estudios Geográficos, 262, 235-265.

SABATÉ, A.; RODRÍGUEZ, J. M. Y DÍAZ, M. A. (1995): Mujeres, espacio y sociedad. Hacia una geografía del género, Madrid, Síntesis.

SALOM, J. Y DELIOS, E. (1998): «La movilidad laboral femenina en el País Valenciano: los factores sociales y laborales en las diferencias por género», Cuadernos de Geografía, 64, 485-511.

SÁNCHEZ, I. (2004): Urbanismo con perspectiva de género. Instituto Andaluz de la Mujer, Junta de Andalucía. Foro Social Europeo. Sevilla. Consulta (20-04-2008). Disponible en www.unidadgenero.com

SEGALES, M. (2007): «Mujeres en movimiento: género, ecología y ciudad». Disponible en http://www.hegoa.ehu.es/congreso/bilbo/komu/4_Mujeres/2_Marcelo-Segales.pdf

SOLÉ, G.; MIRALLES, C. Y CEBOLLADA, A. (2005): «La accesibilidad territorial desde una perspectiva de género». En XIX Congreso de Geógrafos Españoles.

VANDERMISSEN, M.H., THERIAULT, M. Y VILLENEUVE, P. (2005): Work Trips: are there still gender differences? The case of the Quebec Metropolitan Area, 1991 and 2001. TRB 2006 Annual Meeting. 
